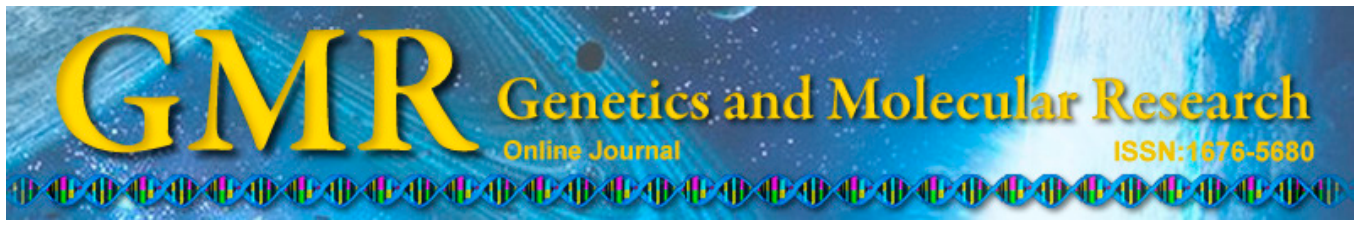

Case Report

\title{
Dynamical evaluation of vaginal micro-ecosystem in a Chinese woman with recurrent vulvovaginal candidiasis
}

\author{
Z.Q. Hu, W. Zhou, X.A. Yue, L.Y. Mu and Y.M. Jiang \\ Department of Laboratory Medicine, West China Second University Hospital, \\ Sichuan University, Chengdu, China \\ Corresponding author: Y.M. Jiang \\ E-mail: yongmeijiang@126.com / jiangyongmei-1@163.com
}

Genet. Mol. Res. 14 (1): 2626-2630 (2015)

Received April 4, 2014

Accepted August 14, 2014

Published March 30, 2015

DOI http://dx.doi.org/10.4238/2015.March.30.22

\begin{abstract}
We reported a rare case of recurrent vulvovaginal candidiasis (RVVC) in this study. Through dynamic evaluation of the vaginal micro-ecosystem, we found that only depuratory degree, spores, blastospores, and hyphae were specific indicators and the "barometer" of RVVC development. Therefore, an understanding of vaginal microecological changes can help clinicians to improve the treatment of patients with RVVC.
\end{abstract}

Key words: Recurrent vulvovaginal candidiasis; Combined treatment; Vaginal micro-ecosystem 


\section{INTRODUCTION}

Vulvovaginal candidiasis (VVC) is a common gynecological disease. More than 50 percent of women older than 25 years have had 1 episode of VVC (Sobel, 2007), but approximately $5 \%$ of these women experience recurrent infection, known as recurrent VVC (RVVC) (Ringdahl, 2006). VVC is mainly caused by Candida infection. Candida can be part of the vaginal ecosystem as a minority germ and is primarily present in the dormant form of spora (Bertholf and Stafford, 1983). A previous study showed that the adhesion germination of Candida is the pathological basis of VVC (Milanese et al., 2008). If the endogenous or exogenous factors of germination are increased, the patient generally shows VVC symptoms. In contrast, if the germination of Candida albicans occurs after adhesion, the acute or recurrent outbreak of carrier women with asymptomatic VVC can be prevented (Raska et al., 2007). RVVC refers to the VVC during which clinical symptoms and signs disappeared after treatment. However, the symptoms can reappear after a negative fungal examination. At least 4 discrete relapses can occur in 1 year and are not related to antibiotic therapy (Ringdahl, 2000).

Dynamic observations of the vaginal micro-ecosystem of RVVC can increase the understanding of the vaginal micro-ecological status and its varied laws as well as to improve treatment plans for RVVC. Thus, we describe a rare case of RVVC caused by $C$. albicans. We evaluated her dynamic vaginal micro-ecological changes to increase the understanding of these changes and assist clinicians in treating RVVC.

\section{CASE REPORT}

A 33-year-old Han Chinese female, married, had been pregnant 3 times and gave birth to 1 child, complained of having Candida vaginitis for more than 2 years with dyspareunia. She visited the outpatient Department of Gynecology recurrently on December 30, 2010; January 10, 2011; February 27, March 1, May 2, and May 17, 2012; and March 26, April 18, June 3 , and June 21, 2013. We collected personal information of the patient from the medical system of our hospital.

She was previously healthy and had no history of other vaginal infectious diseases. She denied having contact with other infectious patients. She also denied her husband having a history of VVC. A recent physical examination on June 3, 2013 indicated that her menstruations typically occurred early, were generous, and showed no menorrhagia. Her vulva was normal without hyperemia or creamy vaginal discharge. No obvious abnormality was revealed by examinations of the uterine body, bilateral appendixes, pelvis, and other systems.

Dynamic evaluations of the vaginal micro-ecosystem included: 1) routine examination, including the depuratory degree, leukocytes, lactobacilli, spores, blastospores, hyphae, Gonorrheal diplococcus, Trichomonas vaginalis, and clue cells; 2 ) flora status, which included flora diversity, bacterial density, dominant flora, and Nugent score; and 3) functional examination, which included $\mathrm{pH}$ value, sialidase, and hydrogen peroxide. Dynamic evaluations of the vaginal micro-ecosystem indicated the occurrence of variations in the depuratory degree, and the spores and blastospores were primly related to the variation of RVVC. The curve of their variation reflected the variation in the vaginal micro-ecosystem. Candidal spores and blastospores were present recurrently. The fungal cultures from February 27 and May 17, 2012 as well as April 18, 2013 were negative, which agreed with fungal microscopic observation. However, the fungal culture was positive on March 26, 2013, which did not agree with the 
fungal microscopic observation. G. diplococcus and T. vaginalis were absent. The entire clue cells, flora diversity, bacterial density, dominant flora, $\mathrm{pH}$, sialidase, hydrogen peroxide, and leukocytes showed recurrent fluctuations. The vaginal micro-ecological changes in this RVVC case are presented in Figure 1.
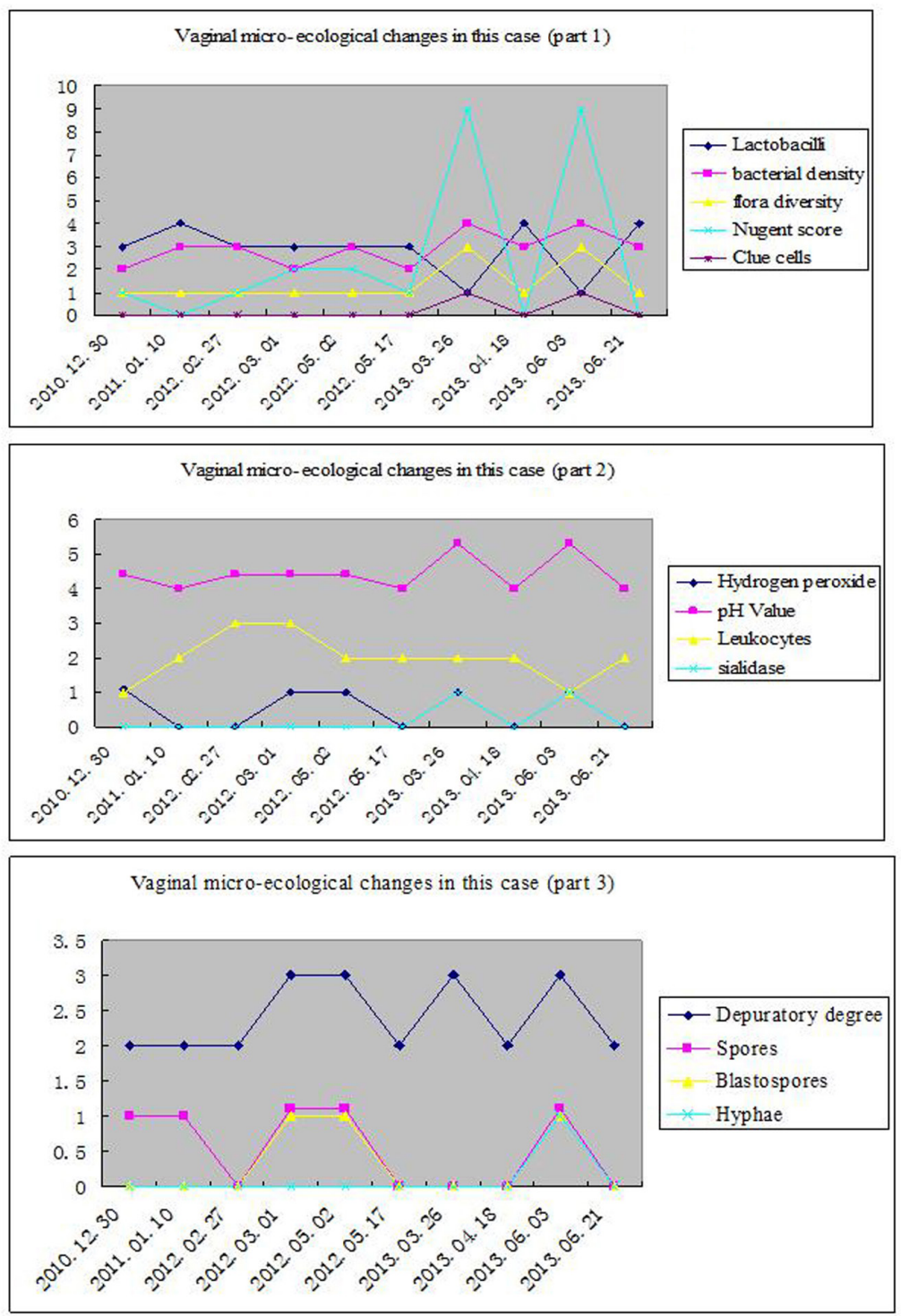

Figure 1. Vaginal micro-ecological changes in our patient. Part 1. Lactobacilli (per oil field): absent $(0),<1(1+)$, 1-5 (2+), 6-30 (3+), >30 (4+); bacterial density (per oil field): absent (0), 0-9 (1+), 10-99 (2+), $100-999(3+)$, clusters (4+); flora diversity (types per oil field): absent (0), 0-3 (1+), 4-6 (2+), 7-9 (3+), $\geq 10(4+)$; clue cells: negative (0), positive (1). Part 2. Hydrogen peroxide and sialidase: negative (0), positive (1); $\mathrm{pH}$ value: 4.0-5.3; leukocyte (per high-power lens): absent $(0),<1(1+), 1-5(2+), 6-30(3+),>30(4+)$. Part 3. Depuratory degree: $1+$ to $4+$; spore, blastospore, and hyphae: absent (0) to present (1). The fungal culture on March 26 was positive. 
According to the repeated complaints from the patient and the results of repeated vaginal micro-ecological evaluation (particularly the results of fungal examination), the patient was diagnosed with RVVC and treated by combined treatment of antifungal drugs, antibacterial vaginosis (BV) drugs, and probiotics on June 3, 2013. The detailed treatment plan was as follows: $100 \mathrm{mg} /$ day fluconazole (Diflucan) capsule, disposable oral intake; $200 \mathrm{mg}$ / night miconazole nitrate suppository, with continued vaginal delivery for 7 days; $0.25 \mathrm{~g} / \mathrm{night}$ living preparation of Lactobacillus, with continued vaginal delivery for 10 days; $2 \mathrm{~g} /$ day tinidazole capsule, continually taken orally; $1 \mathrm{~g} /$ day compound metronidazole effervescent tablets, with continued vaginal delivery for 7 days. After the treatment course and 8 days of drug withdrawal, the patient underwent consultation in the outpatient Department of Gynecology on June 21, 2013.

Further special examination indicated that the vaginal discharge was white and present in a normal amount; the vaginal micro-ecosystem had recovered. The candidal spores, blastospores, and hyphae were absent. Physical examination indicated that the cervix was restored to smooth and the vagina, vulva, uterine body, and bilateral appendixes were normal. No palpable mass in her pelvic and abdominal swelling were touched. Her husband was followed-up and no symptoms were observed. He took prophylactic medication $(100 \mathrm{mg}$ /day fluconazole capsule, take as a draught). There was no cross-infection between the patient and her husband.

\section{DISCUSSION}

We reported this case of RVVC for 3 major reasons. First, the recurrent episodes and difficult treatment of this RVVC patient made the therapeutic process very long. This has become a common problem worldwide and is multifactorial in origin. Many women suffer recurrent episodes, severely affecting the quality of their daily lives (Ramsay et al., 2009). This infection can also greatly affect the physical and mental health and bring about distress to the patient. Second, previous data showed that few studies have been reported in China or other country regarding sustained and dynamic vaginal micro-ecological evaluation of RVVC patients, which may help the treatment of RVVC. This is also the first report of RVCC in Chinese women. Third, some variations in the micro-ecosystem were observed through dynamic vaginal micro-ecological evaluation of this RVVC case.

Through vaginal micro-ecological evaluation, indicators in this patient showed some regular variations. The lactobacilli reached nearly $3+$ to $4+(\geq 6$ per oil field), indicating that the growth of Candida would not inhibit the growth of Lactobacilli. Additionally, Lactobacilli is involved in most of the defense mechanisms in the vagina through concurrent adhesion, producing lactic acid, antimicrobial products, and hydrogen peroxide and through local interactions with the innate and cell-mediated immune systems and plasminogen-plasmin system. The presence of different Lactobacillus species with normal vaginal micro-flora has a strong impact on the stability of the micro-flora and prevents the vagina from other combined infections (Kovachev, 2011). The bacterial density reached $2+$ to $4+(\geq 10$ per oil field), indicating that the vaginal flora was normal during RVVC. The diversity of the vaginal flora was typically single, is frequently dominated by Lactobacillus species (Ma et al., 2012), indicating that multi-flora are rarely present during RVVC. The Nugent score, sialidase, and the clue cells all are BV-specific and not relative to the infection of RVVC. The Nugent score $\geq 7$, the positive result for sialidase, and the presence of clue cells are all present during BV infection. However, abundant clue cells may also cover the presence of the spore, blastospore, and hyphae, which 
may lead to false-negative results in fungal examination. The hydrogen peroxide reflected the function of lactobacillus, the positive or negative of which was not related to the variation of RVVC. The $\mathrm{pH}$ value was typically 4.0-4.5, which was weakly related to RVVC infection and mainly related to lactobacillus variation. This value increased to 5.3 when RVVC was combined with BV. Leukocytes were not a specific indicator of RVVC. The variation in the depuratory degree, the spore, and the blastospore were primly related to RVVC variation. The curve of their variation reflects the dynamic variation of the vaginal micro-ecosystem.

Treatment of RVVC must be individualized based on a comparison of effectiveness, convenience, potential side effects, and cost (Sobel, 2007). The patient described here was subjected to combined treatment with antifungal drugs, anti-BV drugs, and probiotics, based on the principles described above. Interestingly, during the treatment of this case of RVVC, when there was only a single infection of VVC (only spores or blastospores were observed in the vaginal discharge) or a single infection of $\mathrm{BV}$, a dramatic change was not observed. However, during combined infection of RVVC and BV, the patient was cured. No further infection was observed when the patient was followed up.

Through dynamic evaluation of the vaginal micro-ecosystem, we found that only depuratory degree, spores, blastospores, and hyphae were specific indicators and could be considered "barometers" of RVVC development. Therefore, understanding vaginal micro-ecological changes can help clinicians improve treatment of patients with RVVC.

\section{Conflicts of interest}

The authors declare no conflict of interest.

\section{REFERENCES}

Bertholf ME and Stafford MJ (1983). Colonization of Candida albicans in vagina, rectum, and mouth. J. Fam. Practice 16: 919-924.

Kovachev S (2011). Vaginal ecosystem. Akush. Ginekol. 50: 41-49.

Ma B, Forney LJ and Ravel J (2012). Vaginal microbiome: rethinking health and disease. Annu. Rev. Microbiol. 66: 371-389.

Milanese M, Segat L, De Seta F, Pirulli D, et al. (2008). MBL2 genetic screening in patients with recurrent vaginal infections. Am. J. Reprod. Immunol. 59: 146-151.

Ramsay S, Astill N, Shankland G and Winter A (2009). Practical management of recurrent vulvovaginal candidiasis. Sexual Health 14: 18-22.

Raska M, Běláková J, Krupka M and Weigl E (2007). Candidiasis - do we need to fight or to tolerate the Candida fungus? Folia Microbiol. 52: 297-312.

Ringdahl EN (2000). Treatment of recurrent vulvovaginal candidiasis. Am. Fam. Physician 61: 3306-3312.

Ringdahl EN (2006). Recurrent vulvovaginal candidiasis. Mo. Med. 103: 165-168.

Sobel JD (2007). Vulvovaginal candidiasis. Lancet 369: 1961-1971. 